\title{
Antigenic variation in a strain of Trypanosoma brucei transmitted by Glossina morsitans and G. palpalis
}

\author{
By A. R. GRAY \\ The Nigerian Institute for Trypanosomiasis Research,* \\ Vom, Northern Nigeria
}

(Received 5 April 1965)

\begin{abstract}
SUMMARY
Antigenic variation was studied in a strain of Trypanosoma brucei transmitted by Glossina morsitans and G. palpalis. In goats and rabbits infected by tsetse flies, the antigenic character of the strain did not change until the 7 th day of infection; thereafter new antigens developed at 2to 3- day intervals until the infected animal died. The antigens of the $T$. brucei strain developed in a similar sequence in the early stages of infections induced by different tsetse flies; in later stages of the infections, the sequences in which antigens developed varied, but many of those produced in different hosts were similar. A common antigen, provisionally called the basic strain antigen, occurred in all substrains of the strain isolated during the first 7 days of infection from animals infected by different tsetse flies. This basic strain antigen was relatively stable and, when present in trypanosomes ingested by tsetse flies, it persisted throughout the period required for cyclical development and for the remainder of the life of the infective fly. It also tended to displace variant antigens when trypanosomes with such antigens multiplied in environments free from antibody. Tsetse flies which ingested trypanosomes with variant antigens transmitted trypanosomes with either the basic strain antigen only or with a mixture of the ingested variant antigen and the basic strain antigen. The basic strain antigen also developed at an early stage of infection when nonimmune animals were infected with variants of the strain transmitted by syringe. These findings are discussed in relation to the serological classification of brucei subgroup trypanosomes and the immunization of animals against trypanosomiasis.
\end{abstract}

\section{INTRODUCTION}

Antigenic variation in trypanosomes was probably first described by Franke (1905) who found that the serological characteristics of a strain of Trypanosoma equinum changed in an infected host. Subsequent immunological studies (reviewed by Taliaferro (1930) and Soltys (1963)) have confirmed that trypanosomes have a great capacity for antigenic variation, and that both natural strains and clones can produce many different antigens. Certain aspects of antigenic variation in trypanosomes have been studied in considerable detail because of their importance in relation to the development of drug-relapse strains, the immunization of animals against trypanosomiasis and the serological classification of strains. Much has been learned, for example, about the number of antigens which may be produced by a trypanosome strain (Ritz, 1914, 1916; Lourie \& O'Connor, 1937; Osaki, 1959), the

\footnotetext{
* Formerly the West African Institute for Trypanosomiasis Research.
} 
variability of a single stock of a strain in different hosts (Leupold, 1928; Raffel, 1934; Cantrell, 1958; Inoki, 1960), the antigenic stability of relapse strains (Mesnil \& Brimont, 1909; Rosenthal, 1913) and the relation between antigenic variation and the immune response of vertebrate hosts (Massaglia, 1907; Levaditi \& McIntosh, 1910; Mutermilch \& Salamon, 1928; Russell, 1936; Gray, 1962). More recently attempts have been made to characterize variable trypanosomal antigens and to locate their position in the organism (Weitz, 1960; Brown \& Williamson, 1962, 1964; Seed, 1963; Williamson \& Brown, 1964).

Much of this work on antigenic variation in trypanosomes was done with strains maintained by blood-passage in laboratory animals and comparatively little has been done to relate the findings to antigenic variation in strains transmitted by tsetse flies. The antigenic changes which occur in blood-passaged strains probably resemble those that occur in strains transmitted in the field by the interrupted feeding of biting flies of such genera as Tabanus and Stomoxys. Transmission of a trypanosome strain by a tsetse fly, in contrast to mechanical transmission, is effected only after the trypanosomes have completed a cycle of development in the fly, which usually takes at least 12 days, depending on climatic factors and on the species of trypanosome and fly (see Buxton, 1955). The importance of occasional development in tsetse flies in preserving trypanosome strain characteristics such as morphology, pathogenicity and drug-sensitivity has been recognized for many years (Bruce, 1914; Lester, 1932; Murgatroyd \& Yorke, 1937) and it is surprising that the effects of cyclical development on the antigenic characteristics of trypanosomes have been, with one notable exception, largely neglected. Broom \& Brown (1940) studied the serological characters of three strains of Trypanosoma brucei before and after cyclical development in Glossina morsitans and found that serological differences between variants of each strain diminished during development in the flies. Substrains of one strain transmitted by different tsetse flies resembled each other closely, but differed from cyclically transmitted substrains of other strains of the same species.

The work described in the present paper was done to extend the findings of Broom \& Brown because the reduction of serological variants of a strain during development in tsetse flies to an antigenic type which might be characteristic of the strain might be of practical value in the serological classification of strains and in immunization against trypanosomiasis. The experiments were concerned with antigenic variation in a strain of Trypanosoma brucei in animals infected by tsetse flies, and with the effects of cyclical transmission on the antigenic characteristics of substrains and serological variants of the strain.

\section{METHODS}

The strain of Trypanosoma brucei used. The strain was isolated in 1961 from a sheep infected by Glossina morsitans which had been collected near Mokwa, Niger Province, Northern Nigeria. A line of the strain was serially passaged by syringe through fourteen rats before a stock was frozen and stored at $-80^{\circ}$ for use in the present experiments. The strain was polymorphic when recovered from the frozen stock and transmissible by tsetse flies. Rats and mice infected by the injection of such material had acute infections with massive parasitaemias and died after 5-7 days and 3-4 days, respectively. Rats infected with the strain by tsetse flies had 
acute infections and died after 8-10 days, but rabbits and goats had chronic infections with low-grade parasitaemias and died after 4-6 weeks. The period between the bite of an infective fly and the appearance of trypanosomes in the peripheral blood of rats, rabbits, goats and sheep was relatively constant, between 2 and 4 days.

Maintenance of the strain. During experiments, substrains and variants of the trypanosome were maintained for short periods by syringe passage at intervals of 2-3 days in mice. Repeated sub-passage was kept to a minimum and was only used when isolating substrains, when preparing antigens for agglutination tests and when frozen stocks of the strain were being re-established in experimental animals. Stocks of substrains and variants were maintained for longer periods at $-80^{\circ}$ as were antigens for agglutination tests which were prepared as described below.

Experimental animals. White mice (12-20 g.) were used during the isolation and short-term passage of substrains and when preparing antigens for agglutination tests. Rabbits (1-2 kg.), white rats (100-200 g.) and goats and sheep of indigenous West African varieties were used to produce agglutinating antisera, to feed tsetse flies, and in experiments on antigenic variation. Mice, rats and rabbits were drawn from closed laboratory colonies and it was assumed that they were free from trypanosomal infections before experiments were started. Goats and sheep were bought in an area which is free from trypanosomiasis and they were unlikely to be harbouring trypanosomes; however, as a precaution, a wet blood film from each animal was examined daily for 30 days before the animal was used, and samples of blood were inoculated into rats which were then examined daily for trypanosomes for 4 weeks. No infections were ever detected.

Terms used to describe populations of trypanosomes. Several terms were used to describe populations of trypanosomes during the experiments. The strain was a collective term and included all the trypanosomes derived from the population of Trypanosoma brucei isolated from the sheep originally infected by wild-caught Glossina morsitans. The term original strain was used to describe the population of the strain preserved at $-\mathbf{8 0}^{\circ}$ at the beginning of the experiments. Substrains were populations of trypanosomes derived from the original strain or each other; ingested substrains consisted of trypanosomes ingested by tsetse flies when they fed on infected animals; cyclically transmitted substrains consisted of trypanosomes isolated from animals between 3 and 7 days after they were bitten by infective tsetse flies. Serological variants (or simply variants) were substrains shown by serological methods to be antigenically different from other substrains. (Variants in this sense were often called relapse strains in the earlier literature on antigenic variation; since many of the infections studied in the present experiments were not characterized by distinct crises and relapses, although antigenic variation occurred, the term relapse strain has been avoided.)

Methods of counting trypanosomes. For routine purposes, parasitaemias in infected animals were estimated by counting the trypanosomes in sixty microscope fields ( $\times 40$ objective and $\times 8$ eye-piece) of a wet blood film. When accurate estimations of the numbers of trypanosomes in heavy suspensions of organisms were required, a sample of the infected material was suitably diluted with Hayem's solution (Whitby \& Britton, 1953) and the organisms counted in a haemocytometer. 


\section{Infection of tsetse flies with the trypanosome}

Origin and infection of the tsetse flies. Most of the tsetse flies were obtained from Glossina pupae collected in parts of Zaria Province, Northern Nigeria, during the dry season between December and March each year; a few flies were obtained from pupae produced in a laboratory colony of Glossina palpalis. The pupae were incubated in a moist atmosphere at $27-29^{\circ}$ and the flies which emerged were kept individually in glass specimen tubes $(3 \times 1 \mathrm{in}$.), or in groups of twenty of the same sex in wooden Bruce boxes, in a humid atmosphere in a fly-room maintained at 24-26 ${ }^{\circ}$.

Flies were infected by feeding on infected animals on the 1st or 2nd day after they emerged from puparia, since higher infection rates with 'brucei' subgroup trypanosomes are obtained in this way than by feeding flies later in life (Wijers, 1958). In some instances, flies were fed on infected animals for 7-14 days, but in experiments in which the antigens of trypanosomes ingested by individual flies were being compared with the antigens of the trypanosomes which the flies transmitted, they were fed on infected animals once only. After taking infected feeds, flies were maintained on uninfected rats, goats, or sheep until they became infective, were killed or died.

Isolation of infective tsetse fies. Two methods were used to isolate infective tsetse flies from groups of flies which had taken infected feeds.

(a) Infection of a susceptible animal. Towards the end of the period required for cyclical development of the trypanosome (16-18 days after an infected feed), large groups of flies were divided into subgroups of three, four or five individuals which were fed daily on a rat until it became infected. The flies in subgroups which caused infections were then fed on separate rats to identify the infective fly.

(b) Examination of tsetse-fly saliva. This method was occasionally used to find an infective fly in a small group. Hungry flies were induced to attempt to feed on a guinea-pig and a small amount of tsetse-fly saliva was collected by inserting a microscope slide carefully between the proboscis of the fly and the guinea-pig (Burtt, 1946). The saliva was dried in air, fixed with methanol and stained with Giemsa's stain, and examined microscopically for metacyclic trypanosomes.

The number of infective flies isolated. Tsetse flies are difficult to infect with 'brucei' subgroup trypanosomes and the infection rates achieved in the laboratory are usually low. Attempts were made to infect 527 Glossina morsitans and $510 \mathrm{G}$. palpalis with the strain; 18 infective G. morsitans and 3 infective $G$. palpalis were isolated. The period required for cyclical development of the trypanosome in G. morsitans averaged about 22 days, but varied from 19 to 25 days; in G. palpalis the period varied from 29 to 42 days.

\section{Determination of the antigenic relationships of substrains of the trypanosome}

The antigenic relations of substrains and variants of the trypanosome were determined mainly by agglutination tests. In certain instances, indications of antigenic relations from direct agglutination tests were confirmed by absorption tests.

Antigens for agglutination tests. Parasitaemias in infected rabbits and goats were usually scanty, but heavy suspensions of trypanosomes were needed for use as antigens in agglutination tests. When antigens were required, trypanosome substrains were isolated from infected animals by injecting $0.5 \mathrm{ml}$. volumes of blood 
into mice, and sub-passaged from 2 to 5 times at 2 to 3 -day intervals in a series of mice until they caused massive parasitaemias after only 2-3 days of infection. The antigenic characteristics of substrains did not vary when they were passaged in this was provided that the interval between successive sub-passages did not exceed 3-4 days and that the procedure was not repeated too many times. When the substrains were satisfactorily adapted to mice, antigens for agglutination tests were prepared as described by Cunningham \& Vickerman (1962) and stored at $-80^{\circ}$ (Polge \& Soltys, 1957).

Agglutinating antisera. Rabbits and goats infected by tsetse flies produced antibodies which agglutinated trypanosomes. Sera were obtained from such animals at intervals which varied in different experiments, but which were usually between 1 and 3 days. When an antiserum to a particular substrain was needed, a suspension of washed living trypanosomes in Alsever's solution was prepared from blood of infected mice. A rabbit was given a single intravenous injection of $2.0 \mathrm{ml}$. of the suspension (containing 4-8 $\times 10^{8}$ trypanosomes) and bled 6 days later. Such 6 thday antisera had high titres of agglutinins to the antigens of the injected substrains but did not agglutinate other antigens of the strain which developed later as a result of antigenic variation. Antisera were stored at $-20^{\circ}$ until they were required.

Agglutination tests. Agglutination tests were done as described by Cunningham $\&$ Vickerman (1962). The results were read microscopically $(\times 10$ objective and $\times 10$ eye-piece) 25-30 min. after the addition of antigen to diluted antisera. The last dilution of an antiserum which produced a few discrete clumps of trypanosomes was taken as the end-point of the reaction. Good agreement was found between the results of agglutination tests made on different occasions with the same antigen + antibody system and, in general, titrations were repeated once. The titres of agglutinins in antisera are expressed as reciprocals of serum dilutions in all tables showing results of agglutination tests.

Sera were taken from all animals before they were used in experiments and tested for agglutinins to various antigens of the trypanosome strain, but in all cases such pre-infection sera failed to agglutinate trypanosomes.

Absorption tests. Suspensions of trypanosomes with which to absorb antisera were prepared as follows. Groups of rats were infected with appropriate substrains and, 2-3 days later, when the blood contained many trypanosomes, they were anaesthetized and bled out into Alsever's solution. The trypanosomes were separated from the erythrocytes by centrifugation, washed twice with Alsever's solution and resuspended in the same medium. Suspensions were prepared in this way to contain 8-14 $\times 10^{8}$ trypanosomes $/ \mathrm{ml}$.

To absorb agglutinins from an antiserum, $0.7 \mathrm{ml}$. of trypsanosome suspension was mixed with $\mathbf{0 . 1} \mathrm{ml}$. of antiserum in a centrifuge tube and left on the bench for $1 \mathrm{hr}$. The tube was then centrifuged at $3000 \mathrm{rev} . / \mathrm{min}$. for $10 \mathrm{~min}$. with an M.S.E. 'Major' centrifuge (Measuring \& Scientific Equipment, London) and $\mathbf{0 . 2} \mathrm{ml}$. of the clear supernatant fluid removed and mixed with $1.8 \mathrm{ml}$. of trypanosome suspension in another centrifuge tube. After one further hour on the bench, the tube was centrifuged at $3000 \mathrm{rev} . / \mathrm{min}$. for $10 \mathrm{~min}$. and the supernatant fluid separated and placed in a clean tube. A sample of the same antiserum was diluted with saline for control purposes. When testing absorbed sera for residual agglutinins, it was estimated that they had been diluted $1 / 80$ during the absorption procedures. 


\section{RESULTS}

Antigenic variation in the strain of Trypanosoma brucei

in animals infected by tsetse flies

A rabbit and two goats were infected with the trypanosome by single bites of isolated tsetse flies to determine the period between the bite of a fly and the appearance of trypanosomes and antibodies in the blood, and the course of antigenic
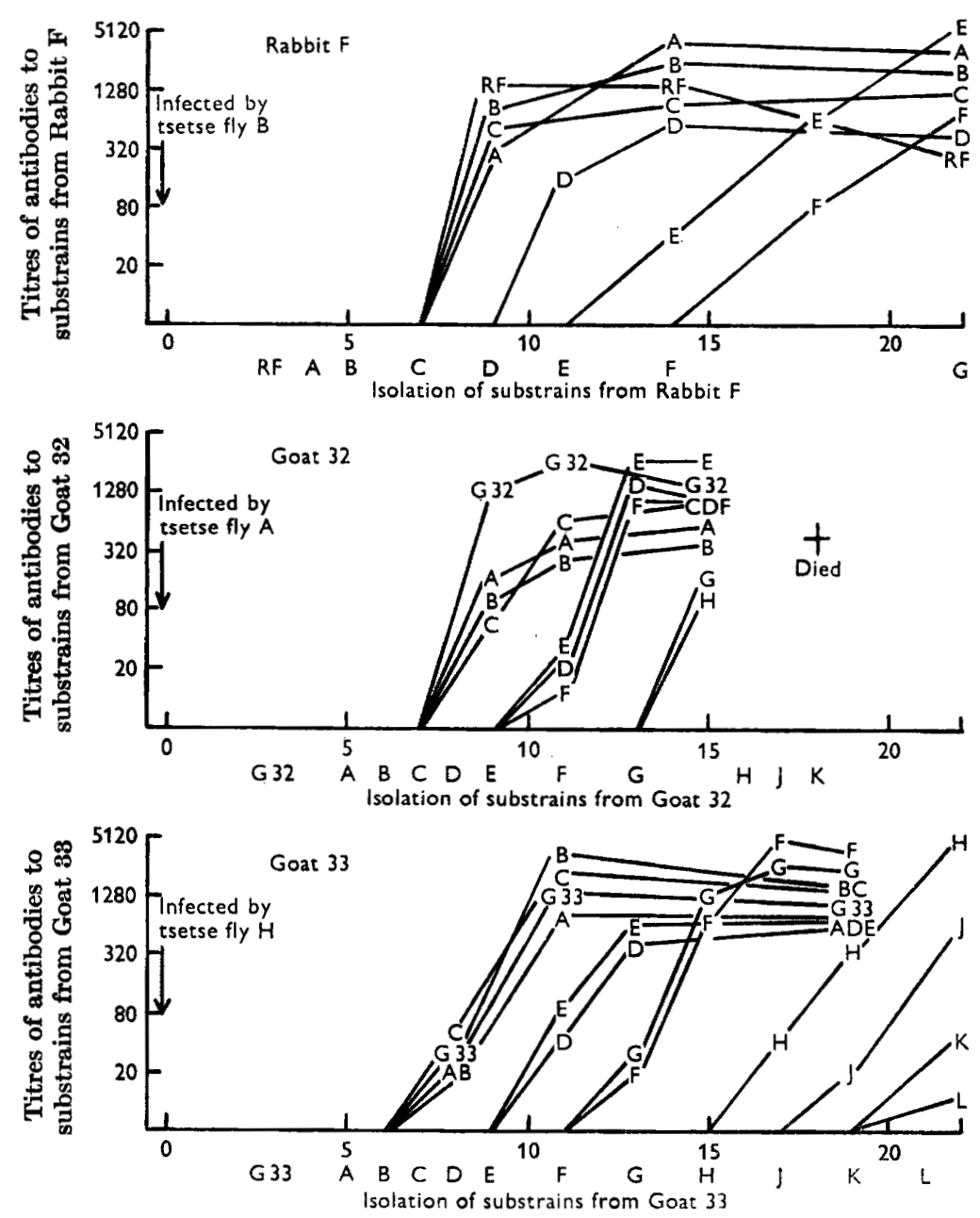

Days after infection

Fig. 1. Antigenic variation in Trypanosoma brucei in animals infected by tsetse flies as shown by agglutinin production to substrains isolated from each animal; similarity of antibody production to groups of substrains indicates their antigenic similarity. A-A, B-B, etc., production of agglutinins to substrains A, B, etc.

variation in the trypanosomes. Substrains of trypanosomes were isolated during the infections at intervals which varied from 1 to $\mathbf{3}$ days. The first substrain isolated from each animal was identified by the number of the animal and those isolated 
subsequently were lettered in sequence. For example, the substrains isolated from Rabbit F were RF, RFA, RFB, RFC and so on to RFG while those from Goat 32 were designated $\mathrm{G} 32, \mathrm{G} 32 \mathrm{~A}, \mathrm{G} \mathrm{32} \mathrm{B}, \mathrm{G} 32 \mathrm{C}, \ldots$ to $\mathrm{G} 32 \mathrm{~K}$. Sera were obtained from the animals before infection and afterwards at 2- to 3-day intervals and tested for agglutinins to antigens prepared from substrains isolated from the same animal.

Sub-inoculation of small volumes of blood into mice showed that trypanosomes were present in the blood of the goats and the rabbit on the 3rd day after the bite of an infective tsetse fly, and agglutination tests showed that antibodies appeared in the sera of these animals on the 8th day of infection (Fig. 1). The antigenic character of the infecting trypanosomes did not change in any instance until the 7 th day of infection because serum taken from each animal on day 9 agglutinated substrains isolated between day 3 and day 7 but not those isolated later. New antigens of the trypanosome strain developed between day 7 and day 9 and the first serologically distinct substrains were isolated from both goats on day 8 and from the rabbit on day 9; thereafter, substrains with different serological characters were isolated at about 3 -day intervals until the animals died.

Reactions between sera and trypanosome substrains indicated that five serological variants were isolated from Rabbit F (represented in experiments described below by substrains RFA, RFD, RFE, RFF and RFG); similarly, four variants were isolated from Goat 32 (represented subsequently by substrains G $32 \mathrm{~A}, \mathrm{G} \mathrm{32} \mathrm{D}$, G $32 \mathrm{G}, \mathrm{G} 32 \mathrm{~K}$ ) and probably 11 variants were isolated from Goat 33 during an infection which lasted for $\mathbf{3 0}$ days.

A comparison of antigens which developed in animals infected by different tsetse flies

The relationships of the antigens of the trypanosome strain which developed in animals infected by different tsetse flies were investigated as follows. Sera were collected at 2-3 day intervals from three goats which had been infected by different tsetse flies, and were tested for agglutinins to five variants of the strain isolated in the previous experiment from Rabbit $F$.

The antigens which developed during the four infections were similar, for sera from the goats agglutinated the rabbit variants (Fig. 2). During the early stages of the infections the antigens tended to develop in a similar sequence. The first trypanosomes which appeared in the blood of the four goats were antigenically similar, because the agglutinins produced by each goat after 9 days of infection reacted strongly with the first rabbit variant (variant $\mathrm{RFA}$ ); 3-4 days later, each goat produced agglutinins to variant RFD, while after a further 3-6 days two of the goats had produced agglutinins to variant RFE (the other goat was dead). Sera taken from the surviving goats 18-24, days after they were bitten by tsetse flies also agglutinated trypanosome variants RFF and RFG.

Although antigens related to those which developed during the rabbit infection also developed during the goat infections, they were probably not the only antigens which developed in the goats. For example, the homologous reactions between sera and variants from Goat 32 indicated that the goat produced antibodies to three variants of the strain before it died (see Fig. 1), but the same goat sera agglutinated only two of the five rabbit variants (Fig. 2).

These findings were confirmed when the experiment was repeated a year later with variants and sera from a different group of animals consisting of three rabbits 
and two goats. Similar antigens developed during the five infections and again, in the early stages of infection, they developed in a similar sequence. However, there was more variation of the period between the bites of infective flies and the first
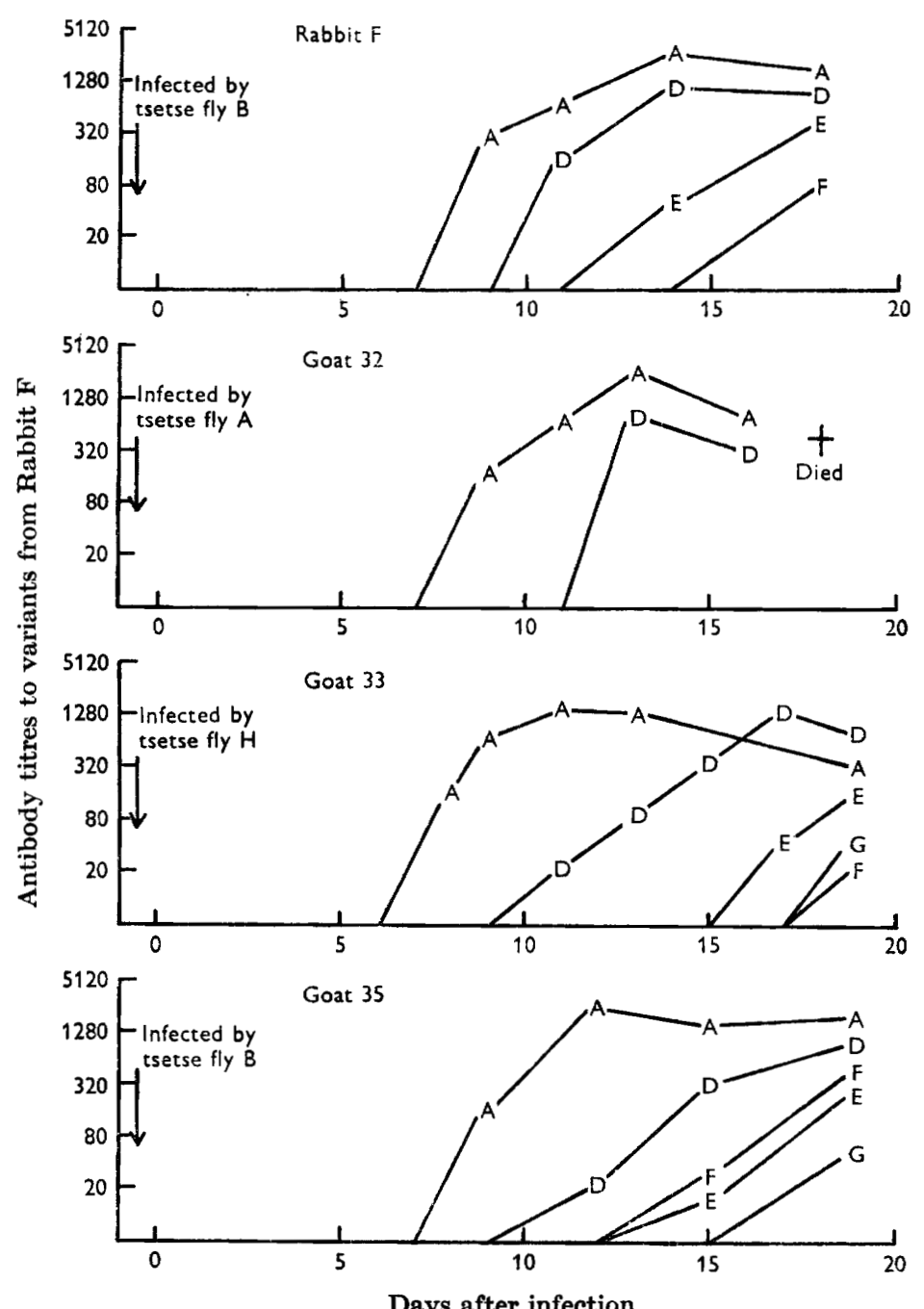

Fig. 2. Comparison of agglutinin production by three goats infected with Trypanosoma brucei by different tsetse flies to five variants of the strain isolated from an infected rabbit, showing that similar antigens develop during different infections. A-A, D-D, E-E, F-F and $G-G$, production of agglutinins to variants $A, D, E, F$ and $G$, respectively.

appearance of antibody in the serum of the animals in this experiment, and the sequences in which the antigens of the strain developed during the later stages of the infections, as shown by the sequences in which antibodies were produced, also varied considerably. Figure 3 shows the variation of the time taken by individual animals to produce agglutinins to a titre of $1 / 160$ to each of the variants isolated from the reference animals in the two experiments. 
The antigenic similarity of substrains transmitted

by different tsetse flies

The results of the preceding experiments suggested that the trypanosomes transmitted by different tsetse flies were antigenically similar. These preliminary observations were extended as follows.

Cyclically transmitted substrains were collected from twenty-one animals which had been infected by different tsetse flies; each substrain was isolated within the first 3-4 days of an infection, before antigenic variation occurred, and therefore probably consisted of trypanosomes of the antigenic type transmitted by the tsetse fly.
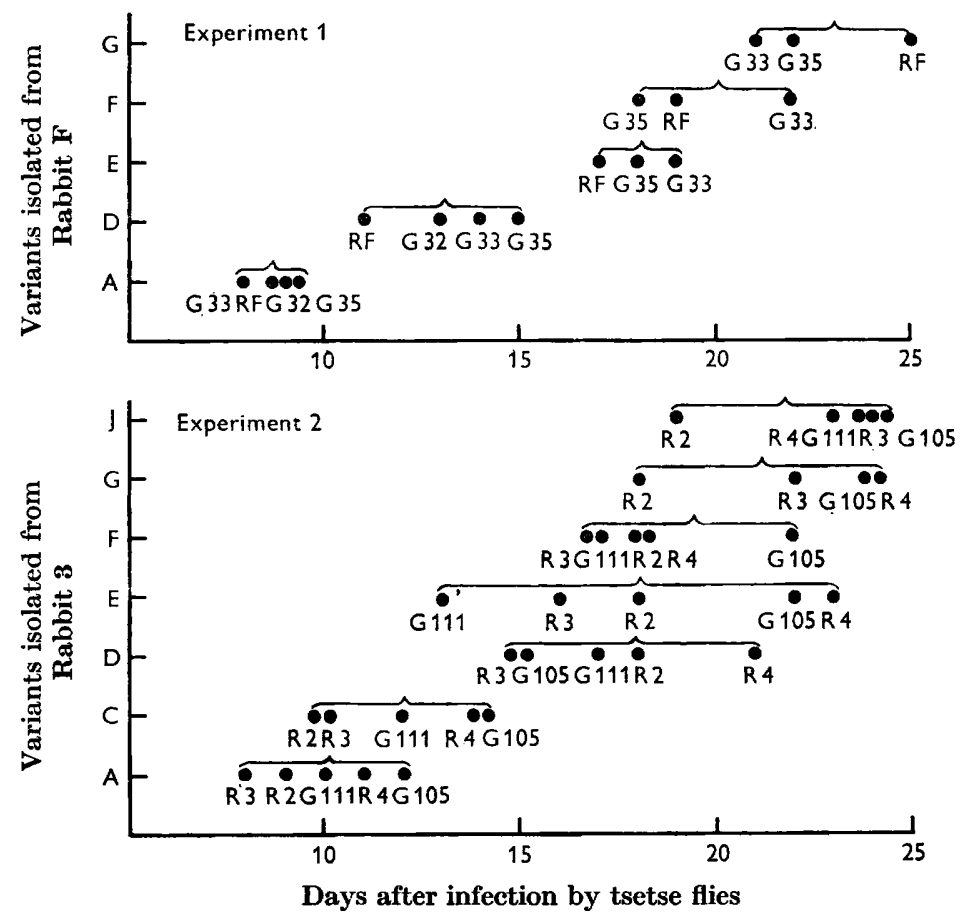

Fig. 3. The sequences in which antigens of Trypanosoma brucei developed in animals infected by different tsetse flies. Variation of the periods which elapsed between infection and agglutinin production to a titre of $1 / 160$ by a rabbit (RF) and three goats (G32, G33, G35) to five variants isolated from the rabbit RF (Experiment 1) compared with variation of agglutinin production by another group of three rabbits $(\mathbf{R 2}, \mathbf{R 3}, \mathbf{R 4})$ and two goats (G105, G111) to variants of the same strain isolated a year later from a different rabbit (R3) (Experiment 2).

Owing to the seasonal availability of tsetse flies, eight of the cyclically transmitted substrains were isolated in 1962 and thirteen in 1963; as the methods used to compare the two groups of substrains differed slightly, the results of the experiments are presented in two sections.

Comparison of substrains transmitted in 1962. The antigenic relationships of the substrains transmitted in $\mathbf{1 9 6 2}$ were determined by direct agglutination tests; a 6th-day antiserum to each substrain was tested for agglutinins to the homologous substrain and to some or all of the other substrains in the group. The substrains 
had antigens in common since, with one exception, an antiserum which had a high titre of agglutinins to its homologous substrain also agglutinated the other substrains. The antiserum to the substrain from Goat 33, which had a high titre of agglutinins to seven of the substrains, was exceptional in that it did not agglutinate the substrain from Rat 4 (Table 1).

Table 1. Titres of agglutinins in antisera to substrains of

Trypanosoma brucei transmitted by tsetse flies in 1962

\begin{tabular}{|c|c|c|c|c|c|c|c|c|c|}
\hline \multirow{2}{*}{$\begin{array}{l}\text { Tsetse fly } \\
\text { which } \\
\text { transmitted } \\
\text { the substrain }\end{array}$} & \multirow{2}{*}{$\begin{array}{l}\text { Substrain } \\
\text { used as } \\
\text { antigen }\end{array}$} & \multicolumn{8}{|c|}{ Titres of agglutinins in antisera to substrain } \\
\hline & & Rat 1 & Rat 2 & G 28 & G 29 & Rat 4 & Rat 5 & Rat 6 & G33 \\
\hline $\mathbf{A}$ & Rat 1 & 1280 & 1280 & 5120 & 160 & 640 & $\mathbf{3 2 0}$ & 320 & 640 \\
\hline $\mathbf{B}$ & Rat 2 & 5120 & 2560 & 5120 & 2560 & 1280 & 2560 & 2560 & 640 \\
\hline $\mathbf{C}$ & G28 & 320 & $\mathbf{3 2 0}$ & 2560 & 640 & 320 & 1280 & 640 & 5120 \\
\hline D & G29 & 640 & 640 & 5120 & 5120 & 640 & 320 & 1280 & 320 \\
\hline $\mathbf{E}$ & Rat 4 & . & . & • & . & 640 & 80 & 160 & - \\
\hline $\mathbf{F}$ & Rat 5 & . & . & . & . & 320 & 1280 & 640 & 1280 \\
\hline $\mathbf{G}$ & Rat 6 & . & . & . & . & 2560 & 2560 & 2560 & 1280 \\
\hline $\mathbf{H}$ & G33 & - & . & . & . & 320 & 640 & 320 & 2560 \\
\hline
\end{tabular}

Table 2. Titres of agglutinins in rabbit antisera to antigens prepared from a substrain of Trypanosoma brucei transmitted by a tsetse fy in 1962 (the reference substrain) and from substrains transmitted by tsetse flies in 1963

\begin{tabular}{|c|c|c|c|c|}
\hline \multirow[b]{2}{*}{$\begin{array}{l}\text { Tsetse fly } \\
\text { which } \\
\text { transmitted } \\
\text { the substrain }\end{array}$} & , & \multirow{2}{*}{$\begin{array}{l}\text { Titres of } \\
\text { agglutinins } \\
\text { in an } \\
\text { antiserum } \\
\text { to the } \\
\text { reference } \\
\text { substrain }\end{array}$} & \multicolumn{2}{|c|}{$\begin{array}{c}\text { Titres of agglutinins in antisera } \\
\text { to the } 1963 \text { substrains }\end{array}$} \\
\hline & $\begin{array}{l}\text { Substrain } \\
\text { used as } \\
\text { antigen }\end{array}$ & & $\begin{array}{c}(a) \\
\text { To the } \\
\text { homologous } \\
\text { substrain }\end{array}$ & $\begin{array}{c}\quad(b) \\
\text { To the } \\
\text { reference } \\
\text { substrain }\end{array}$ \\
\hline $\mathbf{B}$ & The reference substrain & 2560 & na & na \\
\hline 1 & Rat 101 & 160 & 1280 & 2560 \\
\hline 2 & Rat 105 & 80 & 1280 & 320 \\
\hline $\mathbf{3}$ & Rat 109 & 2560 & 1280 & 640 \\
\hline 4 & Rat 113 & 5120 & 5120 & $\mathbf{5 1 2 0}$ \\
\hline 5 & Rat 117 & 5120 & 5120 & 5120 \\
\hline 6. & Rat 121 & 2560 & 2560 & 1280 \\
\hline 8 & Rat 301 & 1280 & 2560 & 2560 \\
\hline 7 & Rat 302 & 2560 & 2560 & 2560 \\
\hline $\mathbf{9}$ & Rat $\mathbf{3 0 3}$ & 1280 & 640 & 1280 \\
\hline 10 & Rat 304 & 2560 & 640 & 2560 \\
\hline 11 & Rat 305 & 5120 & 2560 & 2560 \\
\hline 12 & Rat 306 & 5120 & 2560 & 2560 \\
\hline 13 & Rat 203 & 5120 & 5120 & 5120 \\
\hline
\end{tabular}

Comparison of trypanosome substrains transmitted in 1963. The relationships of the substrains transmitted in 1963 were determined to one of the cyclically transmitted substrains isolated in 1962, first by direct agglutination tests and then by absorption experiments. The substrain isolated from Rat 2 (see Table 1), which had been stored for a year at $-80^{\circ}$, was used as a reference substrain for these purposes. A 6th-day antiserum with a high titre of agglutinins to the reference substrain had a similar high titre to eleven, and a lower titre to two, of the 1963 substrains, indicating 
that they all had antigens in common. This finding was confirmed by tests with antisera to each of the 1963 substrains, which all agglutinated the reference substrain ('Table 2). Antisera to each of the 1963 substrains were then absorbed with a suspension of the reference substrain and the absorbed samples were tested for residual agglutinins to their homologous substrains and to the reference substrain. The absorption tests again confirmed that the 1963 substrains and the reference substrain had an antigen in common and showed that at least three of the 1963 substrains (from Rats 203, 301, 302) possessed an additional antigen because antisera to these substrains still agglutinated their homologous antigens after agglutinins to the reference substrain had been absorbed (Table 3). The antigen which occurred in all the cyclically transmitted substrains isolated in 1963 and in those isolated in 1962, represented by the substrain from Rat 2, was provisionally named the basic strain antigen for the purposes of the following experiments.

Table 3. Effects of absorbing antisera to substrains of Trypanosoma brucei transmitted by tsetse flies in 1963 with a reference substrain transmitted by a tsetse fly in 1962

$\begin{array}{ccccc}\begin{array}{c}\text { Substrain } \\ \text { used as } \\ \text { antigen }\end{array} & \begin{array}{c}\text { Homologous } \\ \text { substrain }\end{array} & \begin{array}{c}\text { Reference } \\ \begin{array}{c}\text { Titres of agglutinins } \\ \text { in antisera diluted } \\ \text { with saline to the }\end{array}\end{array} & \begin{array}{c}\text { Homologous } \\ \text { substrain }\end{array} & \begin{array}{c}\text { Reference } \\ \text { substrain }\end{array} \\ \begin{array}{c}\text { Titres of agglutinins in } \\ \text { antisera absorbed } \\ \text { with the reference } \\ \text { substrain to }\end{array} \\ 101 & \mathbf{3 2 0} & \mathbf{2 5 6 0} & - & - \\ 109 & \mathbf{1 2 8 0} & \mathbf{1 2 8 0} & - & - \\ \mathbf{1 1 3} & \mathbf{2 5 6 0} & \mathbf{5 1 2 0} & - & - \\ \mathbf{3 0 5} & \mathbf{5 1 2 0} & \mathbf{5 1 2 0} & - & - \\ 117 & \mathbf{1 2 8 0} & \mathbf{2 5 6 0} & - & - \\ \mathbf{1 2 1} & \mathbf{5 1 2 0} & \mathbf{5 1 2 0} & - & - \\ \mathbf{2 0 3} & \mathbf{6 4 0} & \mathbf{1 2 8 0} & - & - \\ \mathbf{3 0 1} & \mathbf{2 5 6 0} & \mathbf{2 5 6 0} & \mathbf{2 5 6 0} & - \\ \mathbf{3 0 2} & \mathbf{2 5 6 0} & \mathbf{5 1 2 0} & \mathbf{2 5 6 0} & - \\ \mathbf{3 0 3} & \mathbf{1 2 8 0} & \mathbf{2 5 6 0} & \mathbf{6 4 0} & - \\ \mathbf{3 0 4} & \mathbf{3 2 0} & \mathbf{6 4 0} & - & - \\ \mathbf{3 0 6} & \mathbf{2 5 6 0} & \mathbf{2 5 6 0} & - & - \\ & \mathbf{2 5 6 0} & \mathbf{5 1 2 0} & - & - \\ & & & \end{array}$

The effect of development in the tsetse fly on the antigens of substrains

The antigenic similarity of the cyclically transmitted trypanosome substrains might have been due either to the ingestion of trypanosomes with the basic strain antigen by all the tsetse flies, to alterations of the antigens of serological variants during cyclical development, or to both factors. To investigate these possibilities, attempts were made to infect some tsetse flies with trypanosomes with the basic strain antigen and others with trypanosomes with variant antigens, and to compare the antigens of ingested trypanosomes with those of the organisms the tsetse flies transmitted.

To obtain the material required for these purposes, several batches of 20-30 newly emerged tsetse flies were each fed once on a reservoir animal infected either with one of several serological variants of the trypanosome strain or with a substrain with the basic strain antigen. To guard against the possibility of antigenic 


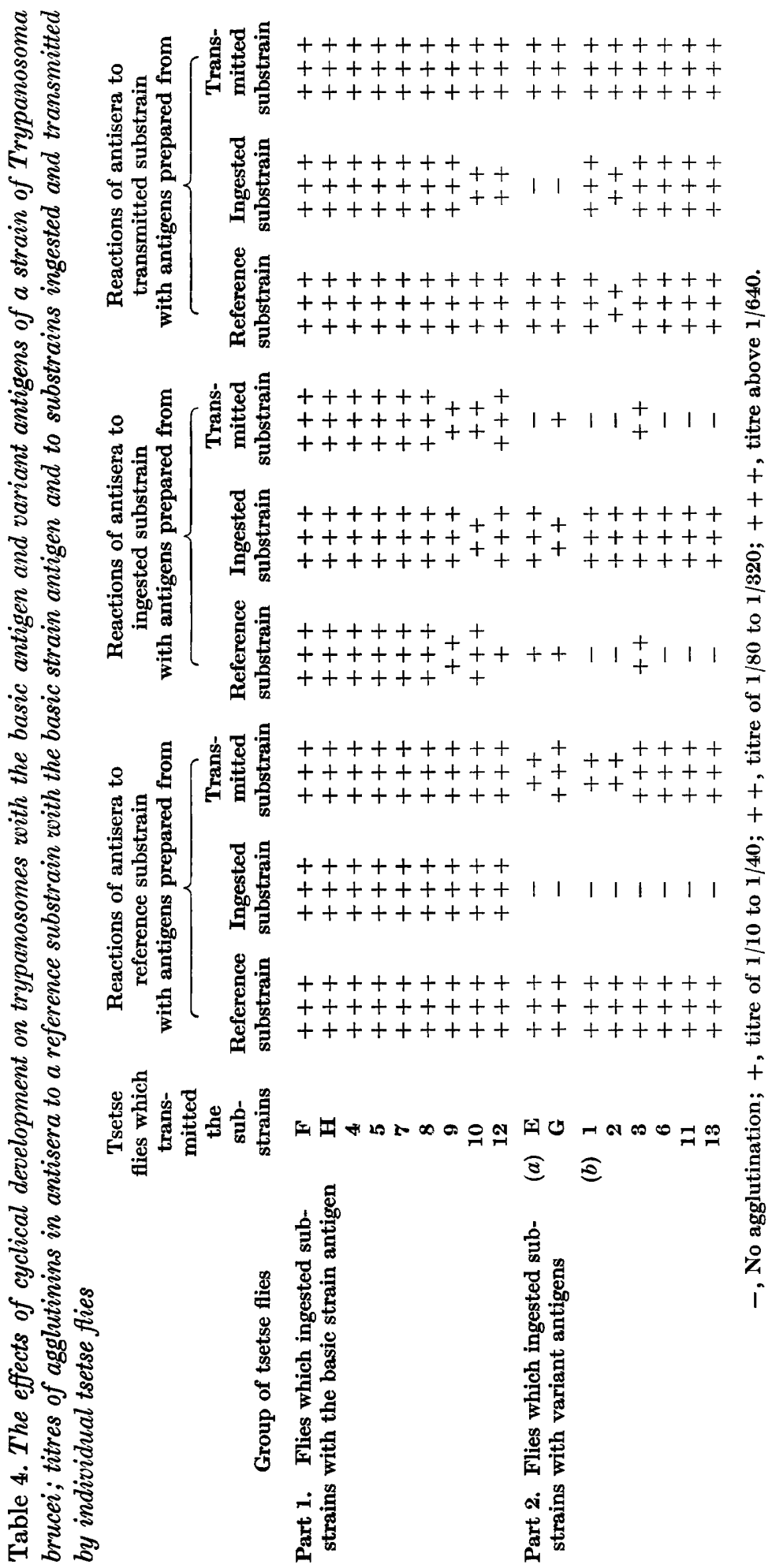


variation in the trypanosomes in the reservoir animals before the flies were fed, an ingested substrain of the antigenic type taken by each batch of flies was isolated from the reservoir host while the flies were being fed, and preserved at $-\mathbf{8 0} 0^{\circ}$. Flies which had taken infected feeds were maintained on uninfected hosts for about 3 weeks while the trypanosomes completed their cycle of development. An infective tsetse fly was then isolated from each batch of flies and used to infect a rat or a goat from which a substrain of the antigenic type transmitted by the fly was subsequently isolated. Antigens for agglutination tests were prepared from the substrains and 6th-day agglutinating antisera were prepared in rabbits. The antigenic relationships of the substrains ingested and transmitted by each tsetse fly were then determined by agglutination tests to each other and to a reference substrain (the substrain from Rat 2) known to possess the basic strain antigen.

The effect of cyclical development on trypanosomes with the basic strain antigen. Nine tsetse flies which had ingested trypanosome substrains with the basic strain antigen became infective. In these instances the ingested substrains were serologically indistinguishable from the reference substrain so that an antiserum to either the reference or the ingested substrain had a similar titre of agglutinins to both substrains. The basic strain antigen persisted during cyclical development in these flies because each cyclically transmitted substrain was agglutinated by antisera to the reference and to the relevant ingested substrain, and an antiserum to each cyclically transmitted substrain had a similar titre to the homologous antigen, the reference substrain and the relevant ingested substrain (Table 4, Part 1).

The effect of cyclical development on trypanosomes with variant antigens. Eight tsetse flies which had ingested serological variants of the trypanosome strain became infective; in these instances the ingested substrains were not agglutinated by the antiserum to the reference substrain. This finding was supported by the reactions of antisera to the ingested substrains, five of which agglutinated only the homologous substrain while the other three had high titres to the homologous substrain and low titres to the reference and cyclically transmitted substrains (Table 4, Part 2).

Two of these tsetse flies, Flies $\mathbf{E}$ and $\mathbf{G}$, transmitted trypanosome substrains which were closely related to the reference substrain and unrelated to the substrains which they ingested. The variant antigens of substrains ingested by these flies were apparently completely replaced by the basic strain antigen because in each case an antiserum to the cyclically transmitted substrain, which had high titres to the homologous and reference substrains, did not agglutinate the ingested substrain (Table 4, Part $2 a$ ).

The other six flies transmitted trypanosome substrains which were related to both the reference and the ingested substrains. In these instances, the variant antigen was only partly replaced by the basic strain antigen during cyclical development, since antisera to the cyclically transmitted substrains had similar titres of agglutinins to the reference, ingested and transmitted substrains (Table 4, Part 2b). Absorption tests confirmed that these six tsetse flies transmitted trypanosomes which possessed a mixture of the basic strain antigen and those of the ingested serological variants. 
The antigenic stability of the trypanosome strain in infective tsetse fies

The following experiment showed that the basic strain antigen responsible for the antigenic similarity of cyclically transmitted trypanosome substrains persisted in tsetse flies from the time they became infective until they died. Cyclically transmitted substrains were isolated from two rats infected by different tsetse flies and stored at $-80^{\circ}$. Seventeen days later, just before they died, the flies infected two more rats from which a second pair of cyclically transmitted substrains was isolated. Sixth-day agglutinating antisera to the pairs of substrains were prepared in rabbits and each antiserum was tested for agglutinins to the homologous substrain and to the substrain from the other rat infected by the same fly. The pairs of substrains were closely related because, in both instances, an antiserum which had a high titre of agglutinins to the substrain isolated from the first rat had a similar titre to the substrain from the second rat (Table 5).

Table 5. Titres of agglutinins in antisera to substrains of Trypanosoma brucei isolated from pairs of animals infected by the same tsetse fly

\begin{tabular}{|c|c|c|c|c|c|}
\hline \multirow{2}{*}{$\begin{array}{l}\text { Tsetse fly } \\
\text { which } \\
\text { transmitted } \\
\text { the } \\
\text { substrain }\end{array}$} & \multirow{2}{*}{$\begin{array}{c}\text { Substrain } \\
\text { used as } \\
\text { antigen }\end{array}$} & \multicolumn{4}{|c|}{ 'Titres of agglutinins in antisera to substrain } \\
\hline & & Rat 1 & Rat 8 & Rat 5 & Rat 14 \\
\hline $\mathbf{A}$ & $\begin{array}{l}\text { Rat } 1 \\
\text { Rat } 3\end{array}$ & $\begin{array}{l}2560 \\
2560\end{array}$ & $\begin{array}{l}\mathbf{3 2 0} \\
\mathbf{8 2 0}\end{array}$ & $\begin{array}{l}\text { na } \\
\text { na }\end{array}$ & $\begin{array}{l}\text { na } \\
\text { na }\end{array}$ \\
\hline $\mathbf{F}$ & $\begin{array}{l}\text { Rat } 5 \\
\text { Rat } 14\end{array}$ & $\begin{array}{l}\text { na } \\
\text { na }\end{array}$ & $\begin{array}{l}\text { na } \\
\text { na }\end{array}$ & $\begin{array}{r}1280 \\
640\end{array}$ & $\begin{array}{l}1280 \\
1280\end{array}$ \\
\hline
\end{tabular}

The predominant character of the basic strain antigen

On several occasions during the preceding experiments, trypanosomes with the basic strain antigen developed at an early stage of infection when animals were infected with serological variants of the strain transmitted by syringe. Antigens with similar properties which occurred in clones of another strain of Trypanosoma brucei have previously been described as predominant antigens (Gray, 1965). The following experiment demonstrated the predominant nature of the basic strain antigen of the strain used in the present experiments. Three rabbits were infected by syringe with variants $R F G, G 32 \mathrm{~K}$ and $\mathrm{G} 33 \mathrm{Q}$, which had been isolated in an earlier experiment at late stages of infection from Rabbit F, Goat 32 and Goat 33, respectively. Sera were collected from the rabbits before infection and afterwards at short intervals for 2-3 weeks. Sera from the rabbit infected with variant RFG were tested for agglutinins to substrain RFA with the basic strain antigen, and to the variants RFD, RFE, RFF and RFG, isolated originally from Rabbit F (see Fig. 1); similarly, sera from the rabbits infected with variants $G 32 \mathrm{~K}$ and $\mathrm{G} 33 \mathrm{Q}$ were tested for agglutinins to substrains with the basic strain antigen and variant antigens isolated from Goat 32 and Goat 33. The first antibodies produced by each of the rabbits agglutinated the variants with which they were infected. The next antibodies produced by the rabbits agglutinated substrains with the basic strain antigen, that is, substrains RFA, G $32 \mathrm{~A}$ and $\mathrm{G} 33 \mathrm{~A}$. In several instances antibodies 
to other antigens of the strain were produced later in the infections, but, in other cases, known strain antigens did not develop during the experiment (Fig. 4). Since the basic strain antigen of the cyclically transmitted trypanosome substrains developed before other known antigens in animals infected by syringe with serological variants, it may be described as a predominant antigen of the strain.
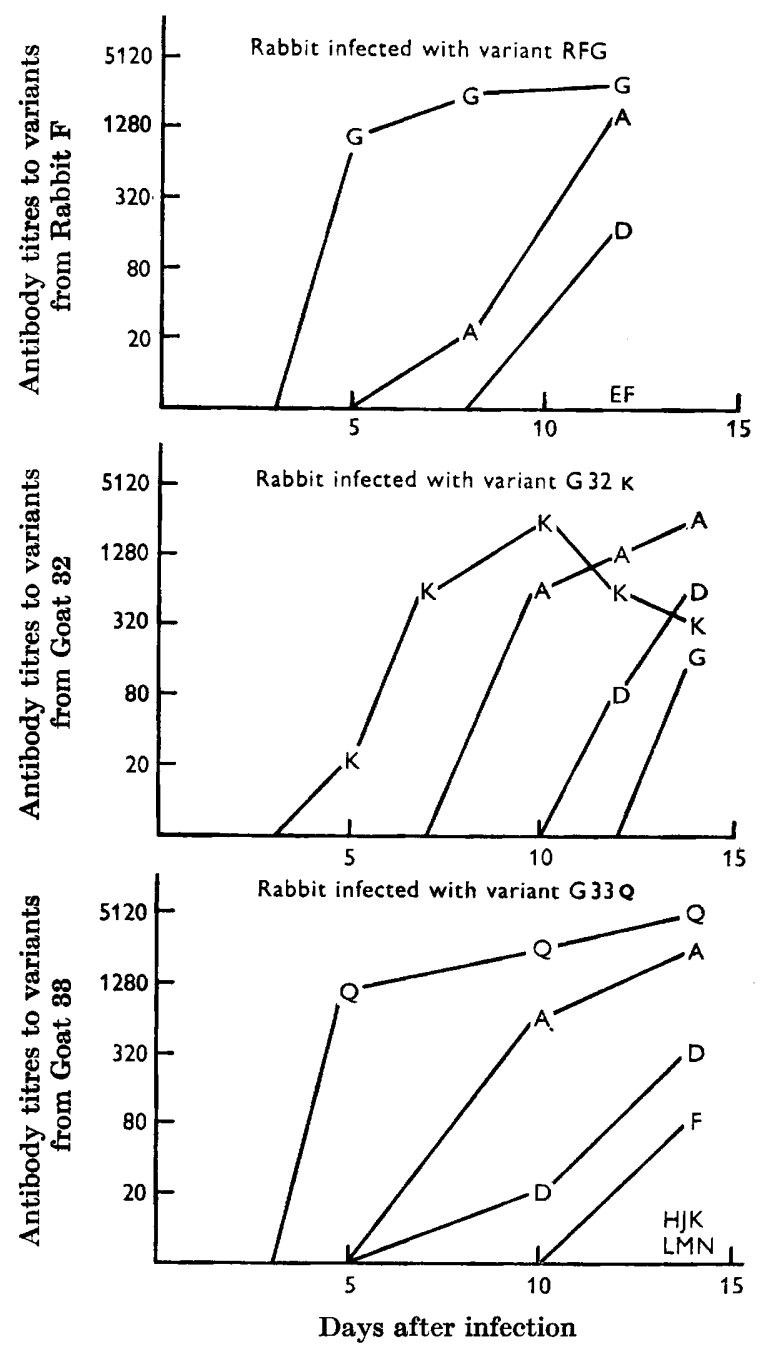

Fig. 4. The predominant character of the basic antigen of a strain of Trypanosoma brucei; agglutinin production by rabbits infected by syringe with serological variants, showing that trypanosomes with the basic strain antigen developed at an early stage of infection. $\mathbf{A}-\mathbf{A}$, antibody to the basic strain antigen; $\mathbf{G}-\mathbf{G}, \mathbf{K}-\mathbf{K}, \mathbf{Q}-\mathbf{Q}, \mathbf{D}-\mathbf{D}$, etc., antibody to variant antigens.

\section{DISCUSSION}

Many features of antigenic variation in Trypanosoma brucei in animals infected by tsetse flies are very similar to those in animals infected with rodent-adapted strains of trypanosomes transmitted by syringe. In addition to their capacity for 
antigenic variation, the trypanosomes of a given strain seem to have an innate tendency to produce one particular antigen which has been provisionally called the basic strain antigen. While investigating the antigenic similarity of cyclically transmitted substrains of a strain, first described by Broom \& Brown (1940) and amply confirmed in the present work, it was found that when trypanosomes with variant antigens were ingested by tsetse flies they were replaced during cyclical development by trypanosomes with the basic antigen. In some instances, ingested variant antigens were completely displaced by the basic strain antigen, but in most cases the change was incomplete, so that the tsetse flies transmitted substrains with a mixture of the ingested variant and the basic strain antigen. The possibility that the degree of reversion of the serological variants to the basic antigenic type of the strain was influenced by the ages of the infections in the flies was unfortunately not investigated, but it may be a factor of considerable importance. The period required for cyclical development of a strain of trypanosomes can vary considerably in different species of tsetse flies and under different experimental conditions. For example, the period required for complete cyclical development of the strain used in the present experiments was about 21 days in Glossina morsitans, while in $G$. palpalis it was at least 29 days. This problem should be considered in future work.

The innate tendency for trypanosomes of the strain to form a characteristic basic strain antigen was also apparent when serological variants were introduced into non-immune hosts by syringe; trypanosomes with the basic strain antigen appeared in the blood of infected animals as soon as antibody to the injected variant was produced. The concept of reversibility of antigenic variation in trypanosomes is not new; earlier workers have noted that variants of a strain sometimes revert to a 'parent' strain type during repeated sub-passage in mice and rats, that is, under conditions aimed at preserving variant characteristies by minimizing the effect of the immune response of the host (see Lourie \& O'Connor, 1937). In this context, Inoki and his co-workers recently determined the proportions of different variants of a strain of Trypanosoma gambiense which developed in mice and found that a high proportion of those trypanosomes which appeared in the early stages of infections induced with different stocks of the strain were of one antigenic type (Osaki, 1959; Inoki, 1960; Inoki, Nakabayashi, Fukukita \& Osaki, 1960).

The reversion of serological variants of a strain to substrains with the basic strain antigen during development in the tsetse fly may be simpler to explain than the host-parasite relationships by which strain characteristics such as morphology, pathogenicity and drug sensitivity are determined during cyclical transmission. Since some tsetse flies transmit trypanosome substrains with a mixture of the ingested variant and the basic strain antigen, it is unlikely that the basic strain antigen develops in association with any of the familiar morphological changes which occur when trypanosomes develop in tsetse flies (Wenyon, 1926), or even with fine ultrastructural changes of the type observed recently by electron microscopy (Vickerman, 1962). It seems more reasonable to suppose that variant antigens are gradually displaced by the basic strain antigen in the absence of host resistance. Although antibodies to some strain antigens are probably ingested by tsetse flies when they take blood meals from animals with chronic infections, and may suppress the development of antigens during the early stages of cyclical development, it is improbable that such antibodies exert any influence for as long as a week, since Weitz \& Buxton (1953) and Cunningham, Harley, Southon \& Lumsden (1962) have shown that blood proteins retain some serological specificity for about 4 days in the 
gut of tsetse flies but are then rapidly denatured. Thus, the environment in which trypanosomes multiply in the tsetse fly is almost certainly free from antibody for at least 2 of the 3 weeks required for cyclical development and may favour the innate tendency of the strain to revert to the basic strain type.

Earlier experiments on antigenic variation have revealed little evidence of organization in the sequence in which the antigens of a strain develop. Experiments on this aspect of the problem have usually been limited to studies of the relationships of the first variants arising from a single stock of a strain in different hosts. Some workers believe that such first-relapse strains of one strain differ greatly (see Lourie \& O'Connor, 1937), but others have found that they belong to a limited number of distinct antigenic types (Leupold, 1928; Osaki, 1959; Inoki, 1960). Few workers have followed the complete course of antigenic variation in experimental animals or compared the sequences in which the antigens of a strain develop in untreated infections in different hosts. Ritz (1916) showed that similar antigens developed in different rabbits infected with a clone of Trypanosoma brucei and Gray (1962), comparing the courses of antigenic variation in rabbits infected with a strain of $T$. brucei, found that the strain antigens developed in a well-defined pattern and that it was possible to predict, within limits, the order in which the antigens would develop from a given stock of the strain. The latter finding indicated that antigenic variation in trypanosomes was not such a random process as earlier work had suggested; this view is supported by results obtained in the present work. When several animals were infected with $T$. brucei by different tsetse flies, similar antigens developed in an identical sequence in the early stages of each infection; at later stages of infections, however, the order in which the antigens were produced varied, but many antigens developing in each infection were identical. In considering the nature of antigenic variation in trypanosomes, its ready reversibility and a degree of organization in the development of the antigens of strains seem to be more in keeping with a process of adaptation in the organisms than with one of mutation and selection of viable mutants, even though Watkins (1964) has produced evidence which seems to favour the latter hypothesis.

Although there is still some doubt about the relative importance of cyclical and mechanical transmission in the spread of African trypanosomiasis in certain areas (Buxton, 1955; Weitz, 1961), it seems that the mode of transmission of a strain is unlikely to be an important factor in controlling its antigenic constitution, since the basic strain antigen and other predominant antigens develop in new hosts infected in either way. It must be emphasized that this view is based on the results of experiments with a strain transferred from one host to another after maximum periods of infection of $\mathbf{3 0}$ days; greater or even permanent antigenic changes might occur in strains in more chronic infections and their antigenic characters might not be controlled by the means described. It would be interesting to see whether the basic strain antigen of a strain is sufficiently distinctive to enable it to be used to trace strains in epidemiological studies of trypanosomiasis.

The implications of these results in relation to the serological classification of trypanosome strains may be mentioned. The antigenic similarity of cyclically transmitted substrains of a strain suggests a basis on which strains of 'brucei' subgroup trypanosomes may be typed. In support of this, Broom \& Brown (1940) and Gray (1963) showed that cyclically transmitted substrains of strains of Trypanosoma brucei from different localities were antigenically distinct but there are at 
least three major difficulties to overcome before such a classification can succeed. Firstly, many strains of ' brucei' subgroup trypanosomes are very difficult to transmit cyclically in the laboratory and the basic strain antigen of a strain can only be identified initially in trypanosomes isolated from an infective tsetse fly; secondly, cyclically transmitted substrains of a strain may possess variant antigens as well as the basic strain antigen and the degree of cross-reaction between variant antigens of different strains is unknown; thirdly, newly isolated strains of 'brucei' subgroup trypanosomes sometimes cause only a low-grade parasitaemia in mice and could not be classified because of difficulties in obtaining enough trypanosomes for agglutination tests.

If further work shows that these difficulties cannot be overcome, it may still be possible to classify trypanosome strains on the basis of their predominant antigens. This method would avoid problems entailed in cyclical transmission and perhaps also the obstacle presented by less virulent strains, because antisera from hosts infected with such strains could be tested for antibody to a comprehensive range of trypanosomes having the basic and predominant antigens of more pathogenic strains. Either method alone, or a combination of both, would be an improvement on current methods of determining antigenic relationships of trypanosomal strains which amount to comparisons of the antigens of single populations of each strain chosen at random from the variety of antigenic forms in which any strain may appear (e.g. Soltys, 1957; Desowitz, 1961; Cunningham \& Vickerman, 1962; Pautrizel, Duret, Tribouley \& Ripert, 1962).

The detection of variant antigens, as well as a basic strain antigen, in cyclically transmitted substrains of a strain casts some doubt on the advisability of attempting to immunize animals against infection by means of trypanosomes possessing the basic strain antigen as suggested by Brown (1963). Immunization might be based more suitably on the bound or somatic antigens of trypanosomes, since such antigens are frequently common to several strains and species, whereas variable antigens have a very narrow range of immunological specificity (Gray, 1961; Weitz, 1962, 1963; Seed, 1963).

I am grateful to Mr T. M. Leach, the acting Director of the Nigerian Institute for Trypanosomiasis Research, and to Dr B. Weitz of the Lister Institute of Preventive Medicine, for their interest and advice during this work. I also wish to thank Dr A. M. Jordan and Mr D. A. T. Baldry for the supply of tsetse-fly pupae. This paper forms part of a thesis approved by the University of London for the degree of Ph.D.

\section{REFERENCES}

Broom, J. C. \& Brown, H. C. (1940). Studies in trypanosomiasis. IV. Notes on the serological characters of Trypanosoma brucei after cyclical development in Glossina morsitans. Trans. roy. Soc. trop. Med. Hyg. 34, 53.

Brown, K. N. (1963). The antigenic character of the Brucei trypanosomes. In Immunity to Protozoa. A Symposium of the British Society for Immunology, p. 204. Oxford: Blackwell Scientific Publications.

Brown, K. N. \& Williamson, J. (1962). Antigens of Brucei trypanosomes. Nature, Lond. 194, 1253.

Brown, K. N. \& Williamson, J. (1964). The chemical composition of trypanosomes. IV. Location of antigens in subcellular fractions of Trypanosoma rhodesiense. Exp. Parasit. 15, 69. 
Bruce, D. (1914). Classification of the African trypanosomes pathogenic to man and domestic animals. Trans. roy. Soc. trop. Med. Hyg. 8, 1.

Burtr, E. (1946). Salivation by Glossina morsitans on to glass slides: a technique for isolating infected flies. Ann. trop. Med. Parasit. 40, 141.

Buxton, P. A. (1955). The Natural History of Tsetse Flies. London: H. K. Lewis Ltd.

CANTRELL, W. (1958). Mutation rate and antigenic variation in Trypanosoma equiperdum. J. infect. Dis. 103, 268.

Cunningham, M. P. \& Vickerman, K. (1962). Antigenic analysis in the Trypanosoma brucei group, using the agglutination reaction. Trans. roy. Soc. trop. Med. Hyg. 56, 48.

Cunningham, M. P., Harley, J. M. B., Southon, H. A. W. \& Lumsden, W. H. R. (1962). Detection of antibodies in blood meals of hematophagous Diptera. Science, 138, 32.

Desowrrz, R. S. (1961). Antigenic relationships between polymorphic and monomorphic strains of the brucei group trypanosomes. J. Immunol. 86, 69.

Franke, E. (1905). Therapeutische Versuche bei Trypanosomenerkrankung. Münch. med. Wschr. 52, (II) 2059. Abstracted in Bull. Sleep. Sickn. Bur. 1909, 1, 218.

Gray, A. R. (1961). Soluble antigens of Trypanosoma vivax and of other trypanosomes. Immunology, 4, 253.

Gray, A. R. (1962). The influence of antibody on serological variation in Trypanosoma brucei. Ann. trop. Med. Parasit. 56, 4.

Gray, A. R. (1963). Antigenic Variation in Some Trypanosomes of the brucei Group. Thesis, University of London.

GraY, A. R. (1965). Antigenic variation in clones of Trypanosoma brucei. I. Immunological relationships of the clones. Ann. trop. Med. Parasit. 59, 27.

INOKI, S. (1960). Studies on antigenic variation in the Welcome strain of Trypanosoma gambiense. II. On the first relapse appearing in mice treated with human plasma. Biken's J. 3, 223.

Inoki, S., Nakabayashi, T., Fukukita, S. \& Osaki, H. (1960). Studies on the immunological variation in Trypanosoma gambiense. V. Antigenic constitution of the relapse type strain in respect to its reversibility to the original type. Biken's $J .3,339$.

LESTER, H. M. O. (1932). The influence of cyclical transmission by Glossina tachinoides on a strain of Trypanosoma brucei made resistant to human serum. Ann. trop. Med. Parasit. 26, 525.

LEUPOLD, F. (1928). Untersuchungen über Rezidivstämme bei Trypanosomen mit Hilfe des Rieckenberg-Phänomens. Z. Hyg. InfektKrankh. 109, 144. Abstracted in Trop. Dis. Bull. 1929, 26, 208.

Levaditi, C. \& McIntosh, J. (1910). Mécanisme de la création de races de trypanosome résistantes aux anticorps. Bull. Soc. Path. exot. 3, 368.

Lourie, E. M. \& O'ConNor, R. J. (1937). A study of Trypanosoma rhodesiense relapse strains in vitro. Ann. trop. Med. Parasit. 31, 319.

Massaglia, A. (1907). Des causes des crises trypanolytiques et des rechutes que les suivent. C. r. hebd. Séanc. Acad. Sci., Paris, 145, 687.

Mesnil, F. \& Brimont, E. (1909). Sur les propriétés protectrices du sérum des animaux trypanosomiés. Races résistantes à ces sérums. Ann. Inst. Past. 23, 129.

Murgatroyd, F. \& Yorke, W. (1937). Studies in chemotherapy. XIV. The stability of drug resistance in trypanosomes. Ann. trop. Med. Parasit. 31, 165.

Mutermilch, S. \& Salamon, E. (1928). Contribution à l'étude du mécanisme de la crise chez cobaye trypanosomié. $C$. $r$. Séanc. Soc. Biol. 98, 348.

Osaki, H. (1959). Studies on the immunological variation in Trypanosoma gambiense (serotypes and mode of relapse). Biken's J. 2, 113.

Pautrizel, R., Duret, J., Tribouley, J. \& Ripert, C. (1962). Etude de la spécificité de la réaction d'agglutination des trypanosomes au cours des trypanosomoses. Bull. Soc. Path. exot. 55, 383.

Polge, C. \& Soltys, M. A. (1957). Preservation of trypanosomes in the frozen state. Trans. roy. Soc. trop. Med. Hyg. 51, 519.

Raffel, S. (1934). Studies in immunity to trypanosomes. I. Acquired immunity in Trypanosoma equiperdum infected rats. The Rieckenberg reaction. Amer. J. Hyg. 19, 416. 
Rrrz, H. (1914). Über Rezidive bei experimenteller Trypanosomiasis. Dtsch. med. Wschr. 40, 1355. Abstracted in Trop. Dis. Bull. 1914, 4, 265.

RrTz, H. (1916). Über Rezidive bei experimenteller Trypanosomiasis. II. Mitteilung. Arch. Schiffs- $u$. Tropenhyg. 20, 397. Abstracted in Trop. Dis. Bull. 1917, 9, 341.

Rosenthal, F. (1913). Untersuchungen über die Genese des Rezidivs bei der experimentellen Trypanosomeninfektion. Z. Hyg. InfektKrankh. 74, 489. Abstracted in Trop. Dis. Bull. 1913, 2, 461.

Russell, H. (1936). Observations on immunity in relapsing fever and trypanosomiasis. Trans. roy. Soc. trop. Med. Hyg. 30, 179.

SEED, J. R. (1963). The characterization of antigens isolated from Trypanosoma rhodesiense. J. Protozool. 10, 380.

Soltys, M. A. (1957). Immunity in trypanosomiasis. II. Agglutination reaction with African trypanosomes. Parasitology, 47, 390.

Soltys, M. A. (1963). Immunity in African trypanosomiasis. Bull. Wld Hlth Org. 28, 753.

Taliaferro, W. H. (1930). The Immunology of Parasitic Infections. London: John Bale, Sons and Danielsson Ltd.

Vickerman, K. (1962). The mechanism of cyclical development in trypanosomes of the Trypanosoma brucei sub-group; an hypothesis based on ultrastructural observations. Trans. roy. Soc. trop. Med. Hyg. 56, 487.

WATKINS, J. F. (1964). Observations on antigenic variation in a strain of Trypanosoma brucei growing in mice. J. Hyg., Camb. 62, 69.

WeITz, B. (1960). The properties of some antigens of Trypanosoma brucei. J. gen. Microbiol. 23, 589.

WeITz, B. (1961). The epidemiology and control of animal trypanosomiasis in Africa. Vet. A. 3, 54.

Werrz, B. (1962). Immunity in trypanosomiasis. In Drugs, Parasites and Hosts. $A$ Biological Council Symposium, p. 180. London: J. \& A. Churchill Ltd.

WerTz, B. (1963). The antigenicity of some African trypanosomes. In Immunity to Protozoa. A Symposium of the British Society for Immunology, p. 196. Oxford: Blackwell Scientific Publications.

Weitz, B. \& Buxton, P. A. (1953). The rate of digestion of blood meals of various haematophagous arthropods as determined by the precipitin test. Bull. ent. Res. 44, 445.

Wenyon, C. M. (1926). Protozoology. London: Baillière, Tindall and Cox.

Whitby, L. E. H. \& Britton, C. J. C. (1953). Disorders of the Blood, 7th ed. London: J. \& A. Churchill Ltd.

WiJers, D. J. B. (1958). Factors that may influence the infection rate of Glossina palpalis with Trypanosoma gambiense. I. The age of the fly at the time of the infected feed. Ann. trop. Med. Parasit. 52, 385.

Williamson, J. \& Brown, K. N. (1964). The chemical composition of trypanosomes. III. Antigenic constituents of Brucei trypanosomes. Exp. Parasit. 15, 44. 